\title{
Stability and Hopf Bifurcation in an HIV-1 Infection Model with Latently Infected Cells and Delayed Immune Response
}

\author{
Haibin Wang and Rui Xu \\ Institute of Applied Mathematics, Shijiazhuang Mechanical Engineering College, No. 97 Heping West Road, \\ Shijiazhuang, Hebei 050003, China \\ Correspondence should be addressed to Haibin Wang; xinerwhb@163.com
}

Received 29 May 2013; Accepted 14 December 2013

Academic Editor: Juan J. Nieto

Copyright (C) 2013 H. Wang and R. Xu. This is an open access article distributed under the Creative Commons Attribution License, which permits unrestricted use, distribution, and reproduction in any medium, provided the original work is properly cited.

An HIV-1 infection model with latently infected cells and delayed immune response is investigated. By analyzing the corresponding characteristic equations, the local stability of each of feasible equilibria is established and the existence of Hopf bifurcations at the CTL-activated infection equilibrium is also studied. By means of suitable Lyapunov functionals and LaSalle's invariance principle, it is proved that the infection-free equilibrium is globally asymptotically stable if the basic reproduction ratio for viral infection $R_{0} \leq 1$; if the basic reproduction ratio for viral infection $R_{0}>1$ and the basic reproduction ratio for CTL immune response $R_{1} \leq 1$, the CTL-inactivated infection equilibrium is globally asymptotically stable. If the basic reproduction ratio for CTL immune response $R_{1}>1$, the global stability of the CTL-activated infection equilibrium is also derived when the time delay $\tau=0$. Numerical simulations are carried out to illustrate the main results.

\section{Introduction}

Mathematical and computational models of the human immune response under HIV-1 infection have received great attention in recent years [1-9]. It is a useful tool of better understanding disease dynamics and making prediction of disease outbreak and evaluations of prevention strategies and drug therapy strategies used against HIV-1 infection.

It is well known that when HIV-1 enters the body, it targets cells with CD4 receptors, including the CD4+ T-cells, the main driver of the immune response. Recent studies have shown that a significant proportion of CD4+ T-cells are infected by the virus, and that this specific population of Tcells might be preferentially infected [10]. In human's immune system, cytotoxic T lymphocytes (CTLs) play an important role in antiviral defense by attacking infected cells. Therefore, it is important and yet has been a hot topic to formulate models to explain the exhaustion of the CD4+ T-cells and CTLs. Such models involve the concentrations of uninfected CD4+ T-cells, $x$, infected CD4+ T-cells that are producing virus, $y$, free virus, $v$, and CTLs, $z$. A basic mathematical model describing HIV-1 infection dynamics that has been studied in $[1,2]$ is of the form

$$
\begin{gathered}
\dot{x}(t)=\lambda-d x(t)-\beta x(t) v(t), \\
\dot{y}(t)=\beta x(t) v(t)-a y(t)-p y(t) z(t), \\
\dot{v}(t)=k y(t)-u v(t), \\
\dot{z}(t)=c y(t)-b z(t),
\end{gathered}
$$

where uninfected, susceptible CD4+ T-cells are created from sources within the body at a rate, $\lambda$, uninfected CD4+ Tcells die at rate $d x(t)$ and become infected at rate $\beta x(t) v(t)$, where $\beta$ is the rate constant describing the infection process; infected cells die at rate $a y(t)$ and are lysed by CTLs at a rate $p y(t) z(t)$; free virus is produced from infected cells at rate $k y(t)$ and is removed at rate $u y(t)$. The CTLs expand at a rate $c y(t)$ and decay at a rate $b z(t)$.

Time delays cannot be ignored in models for immune response, especially the delay between viral appearance and the production of new immune particles [6-9]. Wang et al. [6] studied the effects of the time delay for immune response on a three-dimensional system with $\dot{z}=c y(t-\tau)-b z$. 
By assuming that the production of CTLs also depends on the population of CTL cells, Canabarro et al. [7] investigated the effects of a time delay on a four-dimensional system with $\dot{z}=c y(t-\tau) z(t-\tau)-b z$.

However, in all the previous works mentioned above, they all neglected the fact that once in the cells not all viruses initiate active virus production. A large proportion of CD4+ T-cells are latently infected following the integration of proviral DNA into the host cell genome, some of which can remain quiescent for long periods of time before becoming activated [11]. In [12], such cells are defined as latently infected cells. The capability of the HIV-1 to persist latent inside CD4+ T-cells is currently regarded as a barrier to recovery from infection. But till now, as far as we know, only a few works (see, $[13,14]$ ) concern the effects that latently infected cells are expected to have on HIV-1 infection process. In this paper, motivated by the works of $[1,7,13]$, we modify the basic virus infection model and add a further state variable, $w$, which represents the population of latently infected cells and propose a four-dimensional delayed HIV-1 infection model with latently infected cells. The model is given by

$$
\begin{gathered}
\dot{x}(t)=\lambda-d x(t)-\frac{\beta x(t) y(t)}{1+\alpha y(t)}, \\
\dot{w}(t)=\frac{(1-q) \beta x(t) y(t)}{1+\alpha y(t)}-e w(t)-\delta w(t), \\
\dot{y}(t)=\frac{q \beta x(t) y(t)}{1+\alpha y(t)}-a y(t)+\delta w(t)-p y(t) z(t), \\
\dot{z}(t)=c y(t-\tau) z(t-\tau)-b z(t),
\end{gathered}
$$

where the parameters $\lambda, d, \beta, a, p, c$, and $b$ are the same as those defined in model (1). In our model, we assume that the free virus interacts with the uninfected cells to produce actively infected cells at rate $q \beta x(t) y(t) /[1+\alpha y(t)]$ and latently infected cells at rate $(1-q) \beta x(t) y(t) /[1+\alpha y(t)]$ due to the saturation response of the infection rate, where $0<q<1$ and $\alpha>0$. Latently infected cells containing proviral DNA die at rate $e w(t)$ and become activated at rate $\delta w(t)$. The CTL response is activated at a rate proportional to the number of infected cells at a previous time, $c y(t-\tau) z(t-\tau)$, where $\tau$ is the time delay of CTL response.

The initial conditions for system (2) take the form

$$
\begin{gathered}
x(\theta)=\psi_{1}(\theta), \quad w(\theta)=\psi_{2}(\theta), \\
y(\theta)=\psi_{3}(\theta), \quad z(\theta)=\psi_{4}(\theta), \\
\psi_{1}(\theta) \geq 0, \quad \psi_{2}(\theta) \geq 0, \\
\psi_{3}(\theta) \geq 0, \quad \psi_{4}(\theta) \geq 0, \quad \theta \in[-\tau, 0), \\
\psi_{1}(0)>0, \quad \psi_{2}(0)>0, \quad \psi_{3}(0)>0, \quad \psi_{4}(0)>0,
\end{gathered}
$$

where $\left(\psi_{1}(\theta), \psi_{2}(\theta), \psi_{3}(\theta), \psi_{4}(\theta)\right) \in C\left([-\tau, 0], R_{+0}^{4}\right)$ and the Banach space of continuous functions maps the interval $[-\tau, 0]$ into $R_{+0}^{4}$, where $R_{+0}^{4}=\left\{\left(x_{1}, x_{2}, x_{3}, x_{4}\right): x_{i} \geq 0, i=\right.$ $1,2,3,4\}$.
Our primary goal is to carry out a complete mathematical analysis of system (2) and establish its global dynamics. The organization of this paper is as follows. In Section 2, by analyzing the corresponding characteristic equations, we study the local stability of an infection-free equilibrium and a CTL-inactivated infection equilibrium of model (2). In Section 3, we discuss the local stability and the existence of Hopf bifurcations at the CTL-activated infection equilibrium. In Section 4, by means of suitable Lyapunov functionals and LaSalle's invariance principle, we study the global stability of the infection-free equilibrium and the CTL-inactivated infection equilibrium with any $\tau \geq 0$ and the CTL-activated infection equilibrium with $\tau=0$, respectively. Numerical simulations are carried out in the last section to illustrate the main results. A brief remark is also given in this section to conclude our work.

\section{Equilibria and Their Local Stability}

In this section, we firstly establish the nonnegativity and boundedness of solutions of system (2) and then give sufficient conditions for the existence of each of feasible equilibria of system (2) and discuss the local stability of the infectionfree equilibrium and the CTL-inactivated infection equilibrium, respectively.

Theorem 1. Let $(x(t), w(t), y(t), v(t))$ be any solution of system (2). Then, under the initial conditions (3), all solutions $(x(t), w(t), y(t), v(t))$ are nonnegative on $[0,+\infty)$ and ultimately bounded.

Proof. Since the right hand side of system (2) is completely continuous and locally Lipschitzian on $C$, the solution $(x(t), w(t), y(t), v(t))$ of (2) with initial conditions (3) exists and is unique on $[0, T)$, where $0<T<+\infty$ [15, Chapter 2].

Let us show that $x(t)>0$ for all $t \in[0,+\infty)$. If $x(t)$ were to lose its nonnegativity on some local existence interval $[0, T)$ for some constant $t>0$, there would be a time at $t_{1}>0$. By the first equation of (2), we have $\dot{x}\left(t_{1}\right)=\lambda>0$. That means $x(t)<0$ for $t \in\left(t_{1}-\varepsilon, t_{1}\right)$, where $\varepsilon$ is an arbitrarily small positive constant. This leads to a contradiction. It follows that $x(t)$ is always positive. Further, from the second and the third equation in (2), we have, respectively,

$$
\begin{gathered}
w(t)=w(0) \exp \left\{\int_{0}^{t}\left[\frac{(1-q) \beta x(\sigma) y(\sigma)}{w(\sigma)(1+\alpha y(\sigma))}-(e+\delta)\right] d \sigma\right\}, \\
y(t)=y(0) \exp \left\{\int _ { 0 } ^ { t } \left[\frac{q \beta x(\sigma)}{1+\alpha y(\sigma)}-a\right.\right. \\
\left.\left.+\frac{\delta w(\sigma)}{y(\sigma)}-p z(\sigma)\right] d \sigma\right\},
\end{gathered}
$$

which show that $w(t), y(t)>0$ for all $t \geq 0$. Then by the last equation in (2)

$$
z(t)>z(0) e^{-b t}
$$

confirming that $z(t)>0$ for all $t \geq 0$. 
Next, we prove the ultimate boundedness of (2). Denote

$$
N(t)=x(t)+w(t)+y(t)+\frac{p}{c} z(t+\tau) .
$$

By nonnegativity of the solution, it follows that

$$
\begin{aligned}
\dot{N}(t) & =\lambda-d x-e w-a y-b \cdot \frac{p}{c} z(t+\tau) \\
& \leq \lambda-\min \{a, b, d, e\} N(t) .
\end{aligned}
$$

Then the comparison theorem implies that there exists a $t_{2}>0$ such that $N(t) \leq \lambda / \min \{a, b, d, e\}$, for $t \geq t_{2}$. Let $M_{1}=\max \{\lambda / a, \lambda / b, \lambda / d, \lambda / e\}$; then $N(t) \leq M_{1}$, for $t \geq t_{2}$. This implies that $N(t)$ is ultimately bounded, and so are $x(t)$, $w(t), y(t)$, and $v(t)$. Thus the solutions of (2) are ultimately bounded. This completes the proof.

Clearly, system (2) always has an infection-free equilibrium $E_{0}\left(x_{0}, 0,0,0\right)$, where $x_{0}=\lambda / d$.

Denote

$$
R_{0}=\frac{\beta \lambda(e q+\delta)}{a d(e+\delta)}, \quad R_{1}=\frac{c \beta \lambda(e q+\delta)}{a(e+\delta)[d(c+\alpha b)+\beta b]} .
$$

Here $R_{0}$ and $R_{1}$ are called the basic reproduction ratios for viral infection and CTL immune response of system (2), respectively. It is easy to show that $R_{0}>R_{1}$ always holds. If $R_{0}>1$, system (2) admits a CTL-inactivated infection equilibrium $E_{1}\left(x_{1}, w_{1}, y_{1}, 0\right)$ besides $E_{0}$, where

$$
\begin{gathered}
x_{1}=\frac{\alpha(e q+\delta)+a(e+\delta)}{(e q+\delta)(d \alpha+\beta)}, \quad w_{1}=\frac{a d(1-q)\left(R_{0}-1\right)}{(e q+\delta)(d \alpha+\beta)}, \\
y_{1}=\frac{d\left(R_{0}-1\right)}{d \alpha+\beta} .
\end{gathered}
$$

Furthermore, if $R_{1}>1$, system (2) also admits a CTLactivated infection equilibrium $E^{*}\left(x^{*}, w^{*}, y^{*}, z^{*}\right)$, where

$$
\begin{aligned}
x^{*}=\frac{\lambda(c+\alpha b)}{d(c+\alpha b)+\beta b}, & w^{*}=\frac{\beta \lambda b(1-q)}{(e+\delta)[d(c+\alpha b)+\beta b]}, \\
y^{*}=\frac{b}{c}, & z^{*}=\frac{a}{p}\left(R_{1}-1\right) .
\end{aligned}
$$

Then we give the stability result of $E_{0}$ by the following theorem.

Theorem 2. The infection-free equilibrium $E_{0}\left(x_{0}, 0,0,0\right)$ of system (2) is locally asymptotically stable if $R_{0}<1$ and is unstable if $R_{0}>1$.

Proof. The characteristic equation of system (2) at the infection-free equilibrium $E_{0}\left(x_{0}, 0,0,0\right)$ is of the form

$$
(s+b)(s+d)\left(s^{2}+g_{1} s+g_{0}\right)=0,
$$

where

$$
g_{0}=a(e+\delta)-\frac{(e q+\delta) \beta \lambda}{d}, \quad g_{1}=a+e+\delta-\frac{q \beta \lambda}{d} .
$$

Clearly, (11) always has two negative real roots $s_{1}=-b$ and $s_{2}=-d$. Noting that, if $R_{0}<1,(e q+\delta) \beta \lambda / d<a(e+\delta)$, then $g_{0}>0$. Since $0<q<1,(e q+\delta q) \beta \lambda / d<a(e+\delta)$, that is, $q \beta \lambda / d<a$, then we get that $g_{1}>0$. It is easy to see that (11) has another two negative real roots. Therefore, if $R_{0}<$ 1 , the infection-free equilibrium $E_{0}$ is locally asymptotically stable. If $R_{0}>1, g_{0}<0$, (11) has at least one positive real root. Hence, $E_{0}$ is unstable in this case. The proof of Theorem 2 is completed.

As to the stability of $E_{1}$, we have the following result.

Theorem 3. If $R_{1}<1$, the CTL-inactivated infection equilibrium $E_{1}\left(x_{1}, w_{1}, y_{1}, 0\right)$ is locally asymptotically stable. If $R_{1}>1$, $E_{1}\left(x_{1}, w_{1}, y_{1}, 0\right)$ is unstable.

Proof. The characteristic equation of system (2) at the CTLinactivated infection equilibrium $E_{1}\left(x_{1}, w_{1}, y_{1}, 0\right)$ is of the form

$$
\left(s+b-c y_{1} e^{-s \tau}\right)\left(s^{3}+A s^{2}+B s+C\right)=0,
$$

where

$$
\begin{gathered}
A=a+e+\delta+d+\frac{\beta y_{1}}{1+\alpha y_{1}}+\frac{q \beta x_{1}}{\left(1+\alpha y_{1}\right)^{2}}, \\
B=a(e+\delta)-\frac{\delta \beta x_{1}}{\left(1+\alpha y_{1}\right)^{2}}+d(a+e+\delta)+\frac{(a+e+\delta) \beta y_{1}}{1+\alpha y_{1}} \\
+\frac{2 \beta y_{1}}{1+\alpha y_{1}} \frac{q \beta x_{1}}{\left(1+\alpha y_{1}\right)^{2}}+(e q+2 \delta q+d q) \frac{\beta x_{1}}{\left(1+\alpha y_{1}\right)^{2}}, \\
C=a d(e+\delta)-d \frac{\delta \beta x_{1}}{\left(1+\alpha y_{1}\right)^{2}}+\frac{a(e+\delta) \beta y_{1}}{1+\alpha y_{1}} \\
+2(e+\delta) \frac{q \beta y_{1}}{1+\alpha y_{1}} \frac{\beta x_{1}}{\left(1+\alpha y_{1}\right)^{2}}+(e q+2 \delta q) \frac{d \beta x_{1}}{\left(1+\alpha y_{1}\right)^{2}} .
\end{gathered}
$$

We first consider the following equation

$$
s^{3}+A s^{2}+B s+C=0 .
$$

Obviously, $A>0$. Since

$$
\begin{aligned}
-\frac{\delta \beta x_{1}}{\left(1+\alpha y_{1}\right)^{2}} & >-\frac{\delta \beta x_{1}}{1+\alpha y_{1}}=-\frac{\delta}{y_{1}} \frac{1}{1-q} \frac{(1-q) \beta x_{1} y_{1}}{1+\alpha y_{1}} \\
& =-\frac{\delta}{y_{1}} \frac{1}{1-q}(e+\delta) w_{1} \\
& =-a(e+\delta) \frac{\delta}{e q+\delta}>-a(e+\delta),
\end{aligned}
$$


it is easy to see that $B>0, C>0$. By direct calculation,

$$
\begin{aligned}
& A B-C=d(a+e+\delta)(a+e+\delta+d)+d(a+e+\delta) \\
& \times\left(\frac{\beta y_{1}}{1+\alpha y_{1}}+\frac{q \beta x_{1}}{\left(1+\alpha y_{1}\right)^{2}}\right)+\frac{a^{2} \beta y_{1}}{1+\alpha y_{1}} \\
& +\left(\frac{\beta y_{1}}{1+\alpha y_{1}}+\frac{q \beta x_{1}}{\left(1+\alpha y_{1}\right)^{2}}+(e+\delta+d)\right) \\
& \times \frac{(a+e+\delta) \beta y_{1}}{1+\alpha y_{1}}+\frac{d^{2} q \beta x_{1}}{\left(1+\alpha y_{1}\right)^{2}} \\
& +\left(\frac{\beta y_{1}}{1+\alpha y_{1}}+\frac{q \beta x_{1}}{\left(1+\alpha y_{1}\right)^{2}}+(a+e+\delta)\right) \\
& \times \frac{(e q+2 \delta q+d q) \beta x_{1}}{\left(1+\alpha y_{1}\right)^{2}} \\
& +\left(a+d+\frac{\beta y_{1}}{1+\alpha y_{1}}+\frac{q \beta x_{1}}{\left(1+\alpha y_{1}\right)^{2}}\right) \\
& \times \frac{2 \beta y_{1}}{1+\alpha y_{1}} \frac{q \beta x_{1}}{\left(1+\alpha y_{1}\right)^{2}} \\
& +\left(\frac{\beta y_{1}}{1+\alpha y_{1}}+\frac{q \beta x_{1}}{\left(1+\alpha y_{1}\right)^{2}}+(a+e+\delta)\right) a(e+\delta) \\
& -\left(\frac{\beta y_{1}}{1+\alpha y_{1}}+\frac{q \beta x_{1}}{\left(1+\alpha y_{1}\right)^{2}}+(a+e+\delta)\right) \\
& \times \frac{\delta \beta x_{1}}{\left(1+\alpha y_{1}\right)^{2}}>0 \text {. }
\end{aligned}
$$

Therefore, by Routh-Hurwitz criterion, all roots of (15) have negative real parts.

Then we discuss the location of the roots of the following equation

$$
s+b-c y_{1} e^{-s \tau}=0
$$

Denote $f(s)=s+b-c y_{1} e^{-s \tau}$. If $R_{1}>1$, it is easy to verify

$$
\begin{gathered}
f(0)=\frac{d(c+\alpha b)+\beta b}{d \alpha+\beta}\left(1-R_{1}\right)<0, \\
\lim _{s \rightarrow+\infty} f(s)=+\infty .
\end{gathered}
$$

Hence, $f(s)=0$ has one positive real root. $E_{1}\left(x_{1}, w_{1}, y_{1}, 0\right)$ is unstable.
If $R_{1}<1$, then we prove that all roots of $f(s)=0$ have negative real parts. Assume that $\operatorname{Re} s \geq 0$, then from $f(s)=0$, we derive that

$$
\begin{gathered}
\operatorname{Re} s=b\left[\frac{d(c+\alpha b)+\beta b}{b(d \alpha+\beta)}\left(R_{1}-1\right) e^{-\tau \operatorname{Re} s} \cos (\tau \operatorname{Im} s)\right. \\
\left.+e^{-\tau \operatorname{Re} s} \cos (\tau \operatorname{Im} s)-1\right]<0,
\end{gathered}
$$

which comes to a contradiction. Hence, $\operatorname{Re} s<0$. From the discussion above, we can see that, if $R_{1}<1$, the CTLinactivated infection equilibrium $E_{1}\left(x_{1}, w_{1}, y_{1}, 0\right)$ is locally asymptotically stable. This completes the proof of Theorem 3 .

\section{Stability and Hopf Bifurcation at the CTL-Activated Infection Equilibrium}

From the results above, when $R_{1}>1$, the CTL-inactivated infection equilibrium $E_{1}$ is unstable, and at the same time a CTL-activated infection equilibrium $E^{*}$ emerges. Now we regard $\tau$ as a parameter to study the stability of $E^{*}$ and the existence of Hopf bifurcations.

The characteristic equation of system (2) at the CTLactivated infection equilibrium $E^{*}\left(x^{*}, w^{*}, y^{*}, z^{*}\right)$ is of the form

$$
\begin{aligned}
s^{4}+ & p_{3} s^{3}+p_{2} s^{2}+p_{1} s+p_{0} \\
& -\left(q_{3} s^{3}+q_{2} s^{2}+q_{1} s+q_{0}\right) e^{-s \tau}=0,
\end{aligned}
$$

where

$$
\begin{aligned}
& p_{3}=e+\delta+a+p z^{*}+b+d+\frac{\beta y^{*}}{1+\alpha y^{*}}-\frac{q \beta x^{*}}{\left(1+\alpha y^{*}\right)^{2}}, \\
& p_{2}=(b+e+\delta)\left(d+a+p z^{*}\right)+d\left(a+p z^{*}\right)+b(e+\delta) \\
& +\left(b+e+\delta+a+p z^{*}\right) \frac{\beta y^{*}}{1+\alpha y^{*}} \\
& -(b q+e q+d q+\delta) \frac{\beta x^{*}}{\left(1+\alpha y^{*}\right)^{2}}, \\
& p_{1}=(e+\delta)\left(a+p z^{*}\right)(b+d)+b d\left(e+\delta+a+p z^{*}\right) \\
& -\frac{\beta x^{*}}{\left(1+\alpha y^{*}\right)^{2}}(b+d)(e q+\delta)+\frac{\beta y^{*}}{1+\alpha y^{*}} \\
& \times\left[b\left(a+p z^{*}\right)+(e+\delta)\left(a+p z^{*}\right)+b(e+\delta)\right] \\
& -\frac{b d q \beta x^{*}}{\left(1+\alpha y^{*}\right)^{2}} \\
& p_{0}=b d(e+\delta)\left(a+p z^{*}\right)+\frac{\beta y^{*}}{1+\alpha y^{*}} b(e+\delta)\left(a+p z^{*}\right) \\
& -b d(e q+\delta) \frac{\beta x^{*}}{\left(1+\alpha y^{*}\right)^{2}}
\end{aligned}
$$




$$
\begin{gathered}
q_{3}=b, \\
q_{2}=(e+\delta+a+d) b+\frac{b \beta y^{*}}{1+\alpha y^{*}}-\frac{b q \beta x^{*}}{\left(1+\alpha y^{*}\right)^{2}}, \\
q_{1}=b d(a+e+\delta)+a b(e+\delta)+\frac{b(e+\delta+a) \beta y^{*}}{1+\alpha y^{*}} \\
-\frac{b(e q+d q+\delta) \beta x^{*}}{\left(1+\alpha y^{*}\right)^{2}}, \\
q_{0}=a b d(e+\delta)+a b(e+\delta) \frac{\beta y^{*}}{1+\alpha y^{*}} \\
-b d(e q+\delta) \frac{\beta x^{*}}{\left(1+\alpha y^{*}\right)^{2}} .
\end{gathered}
$$

When $\tau=0$, (21) becomes

$$
s^{4}+\left(p_{3}-q_{3}\right) s^{3}+\left(p_{2}-q_{2}\right) s^{2}+\left(p_{1}-q_{1}\right) s+p_{0}-q_{0}=0 .
$$

It is easy to verify that

$$
p_{0}-q_{0}=b p z^{*}(e+\delta)\left(d+\frac{\beta y^{*}}{1+\alpha y^{*}}\right)>0 .
$$

Since

$$
\begin{aligned}
\frac{q \beta x^{*}}{\left(1+\alpha y^{*}\right)^{2}} & <\frac{q \beta x^{*}}{1+\alpha y^{*}}=\frac{q \beta x^{*} y^{*}}{1+\alpha y^{*}} \frac{1}{y^{*}}=a+p z^{*}-\delta \frac{w^{*}}{y^{*}} \\
& <a+p z^{*},
\end{aligned}
$$

by direct calculation, we can easily derive that

$$
p_{3}-q_{3}=e+\delta+a+p z^{*}+d+\frac{\beta y^{*}}{1+\alpha y^{*}}-\frac{q \beta x^{*}}{\left(1+\alpha y^{*}\right)^{2}}>0 .
$$

Then for $\tau=0$, according to Routh-Hurwitz criterion, all the roots of (21) have negative real parts if and only if the following conditions hold:

(H1)

$$
\begin{gathered}
\left(p_{3}-q_{3}\right)\left(p_{2}-q_{2}\right)-\left(p_{1}-q_{1}\right)>0, \\
\left(p_{3}-q_{3}\right)\left(p_{2}-q_{2}\right)\left(p_{1}-q_{1}\right)-\left(p_{3}-q_{3}\right)^{2}\left(p_{0}-q_{0}\right) \\
-\left(p_{1}-q_{1}\right)^{2}>0 .
\end{gathered}
$$

From what has been discussed above, we have the following result.

Theorem 4. Let $\tau=0$. Then the CTL-activated infection equilibrium $E^{*}\left(x^{*}, w^{*}, y^{*}, z^{*}\right)$ of system (2) is locally asymptotically stable if $R_{1}>1$ and the condition (H1) holds.
For $\tau>0$, it is not easy to find rigorously local stability of $E^{*}$. In the following, we will firstly investigate the existence of purely imaginary roots to (21) following the framework of that in [9].

If $i \omega(\omega>0)$ is a root of (21), separating real and imaginary parts yields

$$
\begin{gathered}
\left(q_{0}-q_{2} \omega^{2}\right) \cos \omega \tau+\left(q_{1} \omega-q_{3} \omega^{3}\right) \sin \omega \tau=\omega^{4}-p_{2} \omega^{2}+p_{0} \\
\left(q_{2} \omega^{2}-q_{0}\right) \sin \omega \tau+\left(q_{1} \omega-q_{3} \omega^{3}\right) \cos \omega \tau=-p_{3} \omega^{3}+p_{1} \omega .
\end{gathered}
$$

Squaring and adding the two equations of (28), we obtain that

$$
\omega^{8}+G_{1} \omega^{6}+G_{2} \omega^{4}+G_{3} \omega^{2}+G_{4}=0,
$$

where

$$
\begin{gathered}
G_{1}=p_{3}^{2}-q_{3}^{2}-2 p_{2}, \\
G_{2}=p_{2}^{2}+2 p_{0}-2 p_{1} p_{3}-q_{2}^{2}+2 q_{1} q_{3}, \\
G_{3}=p_{1}^{2}+2 q_{0} q_{2}-2 p_{0} p_{2}-q_{1}^{2}, \quad G_{4}=p_{0}^{2}-q_{0}^{2} .
\end{gathered}
$$

Let $\mu=\omega^{2}$. Then, from (29), we have that

$$
\mu^{4}+G_{1} \mu^{3}+G_{2} \mu^{2}+G_{3} \mu+G_{4}=0 .
$$

Denote $h(\mu) \equiv \mu^{4}+G_{1} \mu^{3}+G_{2} \mu^{2}+G_{3} \mu+G_{4}$. Then we get

$$
h^{\prime}(\mu)=4 \mu^{3}+3 G_{1} \mu^{2}+2 G_{2} \mu+G_{3} .
$$

Suppose that (31) has positive real roots. Without loss of generality, we assume that it has $n(1 \leq n \leq 4)$ positive real roots, defined by $\mu_{1}<\mu_{2}<\cdots<\mu_{n}$, respectively. Then (29) has $n$ positive real roots

$$
\omega_{1}=\sqrt{\mu_{1}}, \quad \omega_{2}=\sqrt{\mu_{2}}, \ldots, \quad \omega_{n}=\sqrt{\mu_{n}} .
$$

From (28), we have

$$
\begin{aligned}
\cos \omega \tau= & \left(\left(-q_{2} \omega_{0}^{2}+q_{0}\right)\left(\omega_{0}^{4}-p_{2} \omega_{0}^{2}+p_{0}\right)\right. \\
& \left.+\left(-q_{3} \omega_{0}^{3}+q_{1} \omega_{0}\right)\left(-p_{3} \omega_{0}^{3}+p_{1} \omega_{0}\right)\right) \\
& \times\left(\left(-q_{2} \omega_{0}^{2}+q_{0}\right)^{2}+\left(-q_{3} \omega_{0}^{3}+q_{1} \omega_{0}\right)^{2}\right)^{-1} .
\end{aligned}
$$

Therefore, if we denote

$$
\begin{aligned}
\tau_{k}^{(j)}=\frac{1}{\omega_{k}} \arccos [ & \left(\left(-q_{2} \omega_{0}^{2}+q_{0}\right)\left(\omega_{0}^{4}-p_{2} \omega_{0}^{2}+p_{0}\right)\right. \\
& \left.+\left(-q_{3} \omega_{0}^{3}+q_{1} \omega_{0}\right)\left(-p_{3} \omega_{0}^{3}+p_{1} \omega_{0}\right)\right) \\
& \left.\times\left(\left(-q_{2} \omega_{0}^{2}+q_{0}\right)^{2}+\left(-q_{3} \omega_{0}^{3}+q_{1} \omega_{0}\right)^{2}\right)^{-1}\right] \\
& +\frac{2 j \pi}{\omega_{k}},
\end{aligned}
$$

where $k=1,2, \ldots, n ; j=0,1,2, \ldots$, then $\pm i \omega_{k}$ are a pair of purely imaginary roots of (21) with $\tau_{k}^{(j)}$. 
Let $s(\tau)=\alpha(\tau)+i \omega(\tau)$ be the root of (21) near $\tau=$ $\tau_{k}^{(j)}$ satisfying $\alpha\left(\tau_{k}^{(j)}\right)=0, \omega\left(\tau_{k}^{(j)}\right)=\omega_{k}$, then we have the following result.

Theorem 5. Suppose that $\mu_{k}=\omega_{k}^{2}, h^{\prime}\left(\mu_{k}\right) \neq 0$, where $h^{\prime}(\mu)$ is defined by (32). Then

$$
\frac{d\left(\alpha\left(\tau_{k}^{(j)}\right)\right)}{d \tau} \neq 0
$$

and $d\left(\alpha\left(\tau_{k}^{(j)}\right)\right) / d \tau$ and $-h^{\prime}\left(\mu_{k}\right)$ have the same sign.

Proof. Calculating the derivative with respective to $\tau$, we obtain

$$
\begin{aligned}
& \left(4 s^{3}+3 p_{3} s^{2}+2 p_{2} s+p_{1}\right) \frac{d s}{d \tau} \\
& +\left[\tau\left(q_{3} s^{3}+q_{2} s^{2}+q_{1} s+q_{0}\right)-\left(3 q_{3} s^{2}+2 q_{2} s+q_{1}\right)\right] e^{-s \tau} \frac{d s}{d \tau} \\
& =s\left(q_{3} s^{3}+q_{2} s^{2}+q_{1} s+q_{0}\right) e^{-s \tau} .
\end{aligned}
$$

Then we derive from (37) that

$$
\begin{aligned}
\left(\frac{d s}{d \tau}\right)^{-1}= & \frac{-3 s^{4}-2 p_{3} s^{3}-p_{2} s^{2}+p_{0}}{-s^{2}\left(s^{4}+p_{3} s^{3}+p_{2} s^{2}+p_{1} s+p_{0}\right)} \\
& +\frac{2 q_{3} s^{3}+q_{2} s^{2}-q_{0}}{-s^{2}\left(q_{3} s^{3}+q_{2} s^{2}+q_{1} s+q_{0}\right)}-\frac{\tau}{s} .
\end{aligned}
$$

Therefore, we have

$$
\begin{aligned}
{\left[\frac{d(\operatorname{Re} s(\tau))}{d \tau}\right]_{\tau=\tau_{k}^{(j)}}^{-1} } & \\
= & \operatorname{Re}\left[\frac{-3 \omega_{k}^{4}+2 p_{3} i \omega_{k}^{3}+p_{2} \omega_{k}^{2}+p_{0}}{\omega_{k}^{2}\left(\omega_{k}^{4}-p_{3} i \omega_{k}^{3}-p_{2} \omega_{k}^{2}+p_{1} i \omega_{k}+p_{0}\right)}\right] \\
& +\operatorname{Re}\left[\frac{-2 q_{3} i \omega_{k}^{3}-q_{2} \omega_{k}^{2}-q_{0}}{\omega_{k}^{2}\left(-q_{3} i \omega_{k}^{3}-q_{2} \omega_{k}^{2}+q_{1} i \omega_{k}+q_{0}\right)}\right] \\
= & \left(\left(-3 \mu_{k}^{2}+p_{2} \mu_{k}+p_{0}\right)\left(\mu_{k}^{2}-p_{2} \mu_{k}+p_{0}\right)\right. \\
& \left.+2 p_{3} \mu_{k}^{2}\left(p_{1}-p_{3} \mu_{k}\right)\right) \\
& \times\left(\mu_{k}\left(\mu_{k}^{2}-p_{2} \mu_{k}+p_{0}\right)^{2}+\mu_{k}^{2}\left(p_{1}-p_{3} \mu_{k}\right)^{2}\right)^{-1} \\
& +\frac{-2 q_{3} \mu_{k}^{2}\left(q_{1}-q_{3} \mu_{k}\right)+\left(q_{2}^{2} \mu_{k}^{2}-q_{0}^{2}\right)}{\mu_{k}\left(-q_{2} \mu_{k}+q_{0}\right)^{2}+\mu_{k}^{2}\left(q_{1}-q_{3} \mu_{k}\right)^{2}},
\end{aligned}
$$

where $\mu_{k}=\omega_{k}^{2}$. From (29) and (31), we get

$$
\begin{gathered}
\left(\mu_{k}^{2}-p_{2} \mu_{k}+p_{0}\right)^{2}+\mu_{k}\left(p_{1}-p_{3} \mu_{k}\right)^{2} \\
=\left(-q_{2} \mu_{k}+q_{0}\right)^{2}+\mu_{k}\left(q_{1}-q_{3} \mu_{k}\right)^{2}, \\
p_{0}^{2}-q_{0}^{2}=G_{4}=-\left(\mu_{k}^{4}+G_{1} \mu_{k}^{3}+G_{2} \mu_{k}^{2}+G_{3} \mu_{k}\right) .
\end{gathered}
$$

Then we get that

$$
\begin{aligned}
{\left[\frac{d(\operatorname{Re} s(\tau))}{d \tau}\right]_{\tau=\tau_{k}^{(j)}}^{-1} } & \\
= & \left(\left(-3 \mu_{k}^{2}+p_{2} \mu_{k}+p_{0}\right)\left(\mu_{k}^{2}-p_{2} \mu_{k}+p_{0}\right)+2 p_{3} \mu_{k}^{2}\right. \\
& \left.\quad \times\left(p_{1}-p_{3} \mu_{k}\right)-2 q_{3} \mu_{k}^{2}\left(q_{1}-q_{3} \mu_{k}\right)+\left(q_{2}^{2} \mu_{k}^{2}-q_{0}^{2}\right)\right) \\
& \times\left(\mu_{k}\left[\left(-q_{2} \mu_{k}+q_{0}\right)^{2}+\mu_{k}\left(q_{1}-q_{3} \mu_{k}\right)^{2}\right]\right)^{-1} \\
= & \frac{-4 \mu_{k}^{4}-3 G_{1} \mu_{k}^{3}-2 G_{2} \mu_{k}^{2}-G_{3} \mu_{k}}{\mu_{k}\left[\left(-q_{2} \mu_{k}+q_{0}\right)^{2}+\mu_{k}\left(q_{1}-q_{3} \mu_{k}\right)^{2}\right]} \\
= & \frac{-h^{\prime}\left(\mu_{k}\right)}{\left(-q_{2} \mu_{k}+q_{0}\right)^{2}+\mu_{k}\left(q_{1}-q_{3} \mu_{k}\right)^{2}} .
\end{aligned}
$$

Furthermore, it follows that

$$
\begin{aligned}
\operatorname{sign} & {\left[\frac{d(\alpha(\tau))}{d \tau}\right]_{\tau=\tau_{k}^{(j)}} } \\
& =\operatorname{sign}\left[\frac{d(\operatorname{Re} s(\tau))}{d \tau}\right]_{\tau=\tau_{k}^{(j)}} \\
& =\operatorname{sign}\left[\frac{-h^{\prime}\left(\mu_{k}\right)}{\left(-q_{2} \mu_{k}+q_{0}\right)^{2}+\mu_{k}\left(q_{1}-q_{3} \mu_{k}\right)^{2}}\right] .
\end{aligned}
$$

Then, this completes the proof of Theorem 5 .

Applying the Hopf bifurcation theorem for functional differential equation [15], we derive the existence of a Hopf bifurcation at $E^{*}$ as stated in the following theorem.

Theorem 6. Suppose that (31) has at least one simple positive root and $\mu_{0}$ is the last such root. Then there is a Hopf bifurcation for system (2) as $\tau$ passes through $\tau_{0}$ leading to a periodic solution that bifurcates from $E^{*}$, where

$$
\begin{aligned}
\tau_{0}=\frac{1}{\sqrt{\mu_{0}}} \arccos [( & \left.-q_{2} \mu_{0}+q_{0}\right)\left(\mu_{0}^{2}-p_{2} \mu_{0}+p_{0}\right) \\
& \left.+\mu_{0}\left(-q_{3} \mu_{0}+q_{1}\right)\left(-p_{3} \mu_{0}+p_{1}\right)\right) \\
& \left.\times\left(\left(-q_{2} \mu_{0}+q_{0}\right)^{2}+\mu_{0}\left(-q_{3} \mu_{0}+q_{1}\right)^{2}\right)^{-1}\right] \\
& +\frac{2 j \pi}{\sqrt{\mu_{0}}} .
\end{aligned}
$$

Remark 7. As an example, we suppose that (31) has three simple positive roots, denoted by $\mu_{1}<\mu_{2}<\mu_{3}$, respectively. 
Then we have $h^{\prime}\left(\mu_{1}\right)>0, h^{\prime}\left(\mu_{2}\right)<0$, and $h^{\prime}\left(\mu_{3}\right)>0$. By Theorem 5 , it is easy to see that

$$
\begin{array}{r}
\frac{d\left(\alpha\left(\tau_{1}^{(j)}\right)\right)}{d \tau}>0, \quad \frac{d\left(\alpha\left(\tau_{2}^{(j)}\right)\right)}{d \tau}<0, \quad \frac{d\left(\alpha\left(\tau_{3}^{(j)}\right)\right)}{d \tau}>0, \\
j=0,1,2, \ldots,
\end{array}
$$

from which we can conclude that the stability switches may appear as $\tau$ increases.

\section{Global Stability}

In this part, we study the global stability of the infection-free equilibrium and the CTL-inactivated infection equilibrium and then discuss the global stability of the CTL-activated infection equilibrium when $\tau=0$. The strategy of proofs is to use suitable Lyapunov functionals and LaSalle's invariance principle in [16].

Define

$$
F(x)=x-1-\ln x .
$$

Clearly, for $x \in(0,+\infty), F(x)$ is nonnegative and has the global minimum at $x=1$ and $F(1)=0$.

Theorem 8. The infection-free equilibrium $E_{0}\left(x_{0}, 0,0,0\right)$ of system (2) is globally asymptotically stable if $R_{0} \leq 1$.

Proof. Let $(x(t), w(t), y(t), z(t))$ be any positive solution of system (2) with initial conditions (3).

Define

$$
\begin{aligned}
W_{0}(t)= & \frac{1}{2}\left[x(t)-x_{0}\right]^{2}+\frac{\delta}{e q+\delta} w(t)+\frac{e+\delta}{e q+\delta} y(t) \\
& +\frac{p(e+\delta)}{c(e q+\delta)} z(t)+\frac{p(e+\delta)}{e q+\delta} x_{0} \int_{t-\tau}^{t} y(\theta) z(\theta) d \theta .
\end{aligned}
$$

The time derivative of $W_{0}(t)$ along positive solutions of system (2) is given by

$$
\begin{aligned}
\dot{W}_{0}(t)= & {\left[x(t)-x_{0}\right]\left(\lambda-d x(t)-\frac{\beta x(t) y(t)}{1+\alpha y(t)}\right) } \\
& +\frac{\delta}{e q+\delta} x_{0}\left[\frac{(1-q) \beta x(t) y(t)}{1+\alpha y(t)}-e w(t)-\delta w(t)\right] \\
& +\frac{e+\delta}{e q+\delta} x_{0}\left[\frac{q \beta x(t) y(t)}{1+\alpha y(t)}-a y(t)\right. \\
& +\delta w(t)-p y(t) z(t)] \\
& +\frac{p(e+\delta)}{c(e q+\delta)} x_{0}[c y(t-\tau) z(t-\tau)-b z(t)] \\
& +\frac{p(e+\delta)}{e q+\delta} x_{0}[y(t) z(t)-y(t-\tau) z(t-\tau)] .
\end{aligned}
$$

On substituting $\lambda=d x_{0}$ into (47) we derive that

$$
\begin{aligned}
& \dot{W}_{0}(t)=-d\left[x(t)-x_{0}\right]^{2}-\left[x(t)-x_{0}\right] \\
& \times\left[\frac{\beta y(t)}{1+\alpha y(t)}\left(x(t)-x_{0}\right)+\frac{\beta x_{0} y(t)}{1+\alpha y(t)}\right] \\
& +x_{0}\left[\frac{\beta y(t)}{1+\alpha y(t)}\left(x(t)-x_{0}\right)+\frac{\beta x_{0} y(t)}{1+\alpha y(t)}\right. \\
& \left.-\frac{a(e+\delta)}{e q+\delta} y(t)-\frac{p(e+\delta)}{e q+\delta} y(t) z(t)\right] \\
& +\frac{p(e+\delta)}{e q+\delta} x_{0} y(t-\tau) z(t-\tau) \\
& -\frac{p(e+\delta)}{c(e q+\delta)} x_{0} b z(t)+\frac{p(e+\delta)}{e q+\delta} x_{0} y(t) z(t) \\
& -\frac{p(e+\delta)}{e q+\delta} x_{0} y(t-\tau) z(t-\tau) \\
& =-\left(d+\frac{\beta y(t)}{1+\alpha y(t)}\right)\left[x(t)-x_{0}\right]^{2} \\
& -x_{0}\left[-\frac{\beta x_{0} y(t)}{1+\alpha y(t)}+\frac{a(e+\delta)}{e q+\delta} y(t)\right] \\
& -\frac{p(e+\delta)}{c(e q+\delta)} x_{0} b z(t) \\
& =-\left(d+\frac{\beta y(t)}{1+\alpha y(t)}\right)\left[x(t)-x_{0}\right]^{2} \\
& -x_{0} \frac{a(e+\delta)}{e q+\delta} \frac{y(t)}{1+\alpha y(t)}\left[1-R_{0}+\alpha y(t)\right] \\
& -\frac{p(e+\delta)}{c(e q+\delta)} x_{0} b z(t) \text {. }
\end{aligned}
$$

Noting that $R_{0} \leq 1$, by (48), we have that $\dot{W}_{0}(t) \leq 0$. By Theorem 5.3.1 in [15], the solutions of system (2) limit to $M$, the largest invariant subset of $\left\{\dot{W}_{0}(t)=0\right\}$. Clearly, it follows from (48) that $\dot{W}_{0}(t)=0$ if and only if $x=x_{0}$ and $y=z=0$. Noting that $M$ is invariant, for each element in $M$, we have $y=0$, and $\dot{y}(t)=0$. We therefore derive from the third equation of system (2) that

$$
0=\dot{y}(t)=\delta w
$$

which yields $w=0$. Hence, $\dot{W}_{0}(t)=0$ if and only if $(x, w$, $y, z)=\left(x_{0}, 0,0,0\right)$. Accordingly, the global asymptotic stability of $E_{0}$ follows LaSalle's invariance principle. This completes the proof.

We are now in a position to establish the global stability of the CTL-inactivated infection equilibrium $E_{1}$ of system (2).

Theorem 9. The CTL-inactivated infection equilibrium $E_{1}\left(x_{1}\right.$, $\left.w_{1}, y_{1}, 0\right)$ of system (2) is globally asymptotically stable if $R_{0}>1$ and $R_{1} \leq 1$. 
Proof. Let $(x(t), w(t), y(t), z(t))$ be any positive solution of system (2) with initial conditions (3).

\section{Define}

$$
\begin{aligned}
W_{11}(t)= & x_{1} F\left[\frac{x(t)}{x_{1}}\right]+\frac{\delta}{e q+\delta} w_{1} F\left[\frac{w(t)}{w_{1}}\right] \\
& +\frac{e+\delta}{e q+\delta} y_{1} F\left[\frac{y(t)}{y_{1}}\right]+\frac{p(e+\delta)}{c(e q+\delta)} z(t),
\end{aligned}
$$

where the function $F(x)$ is defined in (45).

The derivative of $W_{11}(t)$ along positive solutions of system (2) is given by

$$
\begin{aligned}
\dot{W}_{11}(t)= & \left(1-\frac{x_{1}}{x(t)}\right)\left(\lambda-d x(t)-\frac{\beta x(t) y(t)}{1+\alpha y(t)}\right) \\
& +\frac{\delta}{e q+\delta}\left(1-\frac{w_{1}}{w(t)}\right) \\
& \times\left[\frac{(1-q) \beta x(t) y(t)}{1+\alpha y(t)}-e w(t)-\delta w(t)\right] \\
& +\frac{e+\delta}{e q+\delta}\left(1-\frac{y_{1}}{y(t)}\right) \\
& \times\left(\frac{q \beta x(t) y(t)}{1+\alpha y(t)}-a y(t)+\delta w(t)-p y(t) z(t)\right) \\
& +\frac{p(e+\delta)}{c(e q+\delta)}[c y(t-\tau) z(t-\tau)-b z(t)]
\end{aligned}
$$

On substituting $\lambda=d x_{1}+\beta x_{1} y_{1} /\left(1+\alpha y_{1}\right)$ into (51) we derive that

$$
\begin{aligned}
\dot{W}_{11}(t)= & \left(1-\frac{x_{1}}{x(t)}\right)\left[-d\left(x(t)-x_{1}\right)\right] \\
& +\frac{\beta x_{1} y_{1}}{1+\alpha y_{1}}\left(1-\frac{x_{1}}{x(t)}\right)+\frac{\beta x_{1} y(t)}{1+\alpha y(t)} \\
& +\frac{\delta}{e q+\delta}\left[-\frac{(1-q) \beta x(t) y(t)}{1+\alpha y(t)} \frac{w_{1}}{w(t)}+(e+\delta) w_{1}\right] \\
& -\frac{e+\delta}{e q+\delta} a y(t)-\frac{e+\delta}{e q+\delta} p y(t) z(t) \\
& +\frac{e+\delta}{e q+\delta}\left(-\frac{q \beta x(t) y(t)}{1+\alpha y(t)} \frac{y_{1}}{y(t)}+a y_{1}-\delta w(t) \frac{y_{1}}{y(t)}\right. \\
& +\frac{e+\delta}{e q+\delta} p y(t-\tau) z(t-\tau)-\frac{p b(e+\delta)}{c(e q+\delta)} z(t) .
\end{aligned}
$$

Noting that

$$
\begin{gathered}
a y_{1}=\frac{q \beta x_{1} y_{1}}{1+\alpha y_{1}}+\delta w_{1}, \quad \frac{(1-q) \beta x_{1} y_{1}}{1+\alpha y_{1}}=(e+\delta) w_{1}, \\
y_{1}=\frac{\beta \lambda(e q+\delta)-a d(e+\delta)}{a(e+\delta)(d \alpha+\beta)},
\end{gathered}
$$

we derive from (52) that

$$
\begin{aligned}
& \dot{W}_{11}(t)=-\frac{d\left[x(t)-x_{1}\right]^{2}}{x(t)}+\frac{\beta x_{1} y_{1}}{1+\alpha y_{1}}\left(1-\frac{x_{1}}{x(t)}\right) \\
& +\frac{\beta x_{1} y(t)}{1+\alpha y(t)}-\frac{e+\delta}{e q+\delta} a y(t)-\frac{e+\delta}{e q+\delta} p y(t) z(t) \\
& -\frac{\delta}{e q+\delta} \frac{(1-q) \beta x(t) y(t)}{1+\alpha y(t)} \frac{w_{1}}{w(t)} \\
& +\frac{\delta}{e q+\delta} \frac{(1-q) \beta x_{1} y_{1}}{1+\alpha y_{1}} \\
& -\frac{e+\delta}{e q+\delta} \frac{q \beta x(t) y(t)}{1+\alpha y(t)} \frac{y_{1}}{y(t)} \\
& +\frac{e+\delta}{e q+\delta}\left(\frac{q \beta x_{1} y_{1}}{1+\alpha y_{1}}+\delta w_{1}\right) \\
& -\frac{e+\delta}{e q+\delta} \delta w_{1} \frac{w(t)}{w_{1}} \frac{y_{1}}{y(t)} \\
& +\frac{e+\delta}{e q+\delta} p y_{1} z(t)-\frac{p b(e+\delta)}{c(e q+\delta)} z(t) \\
& +\frac{e+\delta}{e q+\delta} p y(t-\tau) z(t-\tau) \\
& =-\frac{d\left[x(t)-x_{1}\right]^{2}}{x}-\frac{e+\delta}{e q+\delta} p y(t) z(t) \\
& +\frac{e+\delta}{e q+\delta} p y(t-\tau) z(t-\tau)+\frac{\delta}{e q+\delta} \frac{(1-q) \beta x_{1} y_{1}}{1+\alpha y_{1}} \\
& \times\left(4-\frac{x_{1}}{x(t)}-\frac{w_{1}}{w(t)} \frac{x(t) y(t)}{x_{1} y_{1}} \frac{1+\alpha y_{1}}{1+\alpha y(t)}\right. \\
& \left.-\frac{y_{1} w(t)}{w_{1} y(t)}-\frac{1+\alpha y(t)}{1+\alpha y_{1}}\right)+\frac{e+\delta}{e q+\delta} \frac{q \beta x_{1} y_{1}}{1+\alpha y_{1}} \\
& \times\left(3-\frac{x_{1}}{x(t)}-\frac{x(t)}{x_{1}} \frac{1+\alpha y_{1}}{1+\alpha y(t)}-\frac{1+\alpha y(t)}{1+\alpha y_{1}}\right) \\
& -\frac{\alpha \beta x_{1}\left[y(t)-y_{1}\right]^{2}}{\left(1+\alpha y_{1}\right)^{2}[1+\alpha y(t)]} \\
& +\frac{p(e+\delta)[d(c+\alpha b)+\beta b]}{c(e q+\delta)(d \alpha+\beta)}\left(R_{1}-1\right) z(t) \text {. }
\end{aligned}
$$


Define

$$
W_{1}(t)=W_{11}(t)+\frac{p(e+\delta)}{e q+\delta} \int_{t-\tau}^{t} y(\theta) z(\theta) d \theta .
$$

Then we have

$$
\begin{aligned}
\dot{W}_{1}(t)= & \dot{W}_{11}(t)+\frac{p(e+\delta)}{e q+\delta}[y(t) z(t)-y(t-\tau) z(t-\tau)] \\
= & -\frac{d\left(x(t)-x_{1}\right)^{2}}{x(t)}+\frac{\delta}{e q+\delta} \frac{(1-q) \beta x_{1} y_{1}}{1+\alpha y_{1}} \\
& \times\left(4-\frac{x_{1}}{x(t)}-\frac{w_{1}}{w(t)} \frac{x(t) y(t)}{x_{1} y_{1}} \frac{1+\alpha y_{1}}{1+\alpha y(t)}-\frac{y_{1} w(t)}{w_{1} y(t)}\right. \\
& \left.-\frac{1+\alpha y(t)}{1+\alpha y_{1}}\right)+\frac{e+\delta}{e q+\delta} \frac{q \beta x_{1} y_{1}}{1+\alpha y_{1}} \\
& \times\left(3-\frac{x_{1}}{x(t)}-\frac{x(t)}{x_{1}} \frac{1+\alpha y_{1}}{1+\alpha y(t)}-\frac{1+\alpha y(t)}{1+\alpha y_{1}}\right) \\
& -\frac{\alpha \beta x_{1}\left[y(t)-y_{1}\right]^{2}}{\left(1+\alpha y_{1}\right)^{2}[1+\alpha y(t)]} \\
& +\frac{p(e+\delta)[d(c+\alpha b)+\beta b]}{c(e q+\delta)(d \alpha+\beta)}\left(R_{1}-1\right) z(t) .
\end{aligned}
$$

Noting that $x_{1}, w_{1}, y_{1}>0, R_{0}>1$, and $R_{1} \leq 1$, by (56), we have that $\dot{W}_{1}(t) \leq 0$. By Theorem 5.3.1 in [15], the solutions of system (2) limit to $M$, the largest invariant subset of $\left\{\dot{W}_{1}(t)=\right.$ $0\}$. It is readily seen from (56) that $\dot{W}_{1}(t)=0$ holds if and only if $(x, w, y, z)=E_{1}\left(x_{1}, w_{1}, y_{1}, 0\right)$. Using a similar argument as that in the proof of Theorem 8 and by LaSalle's invariance principle, the global asymptotic stability of $E_{1}$ follows. This completes the proof.

Finally, we give the global stability result of $E^{*}$ when there is no time delay, that is, $\tau=0$.

Theorem 10. Let $\tau=0$. The CTL-activated infection equilibrium $E^{*}\left(x^{*}, w^{*}, y^{*}, z^{*}\right)$ of system (2) is globally asymptotically stable if $R_{1}>1$.

Proof. Define

$$
\begin{aligned}
W^{*}(t)= & x^{*} F\left(\frac{x}{x^{*}}\right)+\frac{\delta}{e q+\delta} w^{*} F\left(\frac{w}{w^{*}}\right) \\
& +\frac{e+\delta}{e q+\delta} y^{*} F\left(\frac{y}{y^{*}}\right)+\frac{p(e+\delta)}{c(e q+\delta)} z^{*} F\left(\frac{z}{z^{*}}\right),
\end{aligned}
$$

where the function $F(x)$ is defined in (45).

Calculating the derivative of $W^{*}(t)$ along positive solutions of system (2), it follows that

$$
\begin{aligned}
\dot{W}^{*}(t)= & \left(1-\frac{x^{*}}{x}\right)\left(\lambda-d x-\frac{\beta x y}{1+\alpha y}\right) \\
& +\frac{\delta}{e q+\delta}\left(1-\frac{w^{*}}{w}\right)\left[\frac{(1-q) \beta x y}{1+\alpha y}-e w-\alpha w\right] \\
& +\frac{e+\delta}{e q+\delta}\left(1-\frac{y^{*}}{y}\right)\left(\frac{q \beta x y}{1+\alpha y}-a y+\delta w-p y z\right) \\
& +\frac{p(e+\delta)}{c(e q+\delta)}\left(1-\frac{z^{*}}{z}\right)(c y z-b z) .
\end{aligned}
$$

On substituting $\lambda=d x^{*}+\beta x^{*} y^{*} /\left(1+\alpha y^{*}\right)$ into (58) we derive that

$$
\begin{aligned}
\dot{W}^{*}(t)= & \left(1-\frac{x^{*}}{x}\right)\left[-d\left(x-x^{*}\right)+\frac{\beta x^{*} y^{*}}{1+\alpha y^{*}}\right]+\frac{\beta x^{*} y}{1+\alpha y} \\
& +\frac{\delta}{e q+\delta}\left[-\frac{(1-q) \beta x y}{1+\alpha y} \frac{w^{*}}{w}+(e+\alpha) w^{*}\right] \\
& -\frac{e+\delta}{e q+\delta} a y+\frac{e+\delta}{e q+\delta} \\
& \times\left[-\frac{q \beta x y}{1+\alpha y} \frac{y^{*}}{y}+a y^{*}-\delta w \frac{y^{*}}{y}+p y^{*} z\right] \\
& +\frac{p(e+\delta)}{c(e q+\delta)}\left(-b z-c y z^{*}+b z^{*}\right) .
\end{aligned}
$$

At the steady state $E^{*}$,

$$
\begin{aligned}
(e+\delta) w^{*} & =\frac{(1-q) \beta x^{*} y^{*}}{1+\alpha y^{*}}, \\
a y^{*}+p y^{*} z^{*} & =\frac{q \beta x^{*} y^{*}}{1+\alpha y^{*}}+\delta w^{*}, \\
c y^{*} & =b .
\end{aligned}
$$

Then it follows from (59) that

$$
\begin{aligned}
\dot{W}^{*}(t)= & -\frac{d\left(x-x^{*}\right)^{2}}{x}+\frac{\beta x^{*} y^{*}}{1+\alpha y^{*}}\left(1-\frac{x^{*}}{x}\right)+\frac{\beta x^{*} y}{1+\alpha y} \\
& -\frac{e+\delta}{e q+\delta} a y-\frac{\delta}{e q+\delta} \frac{(1-q) \beta x y}{1+\alpha y} \frac{w^{*}}{w} \\
& +\frac{\delta}{e q+\delta} \frac{(1-q) \beta x^{*} y^{*}}{1+\alpha y^{*}}-\frac{e+\delta}{e q+\delta} \frac{q \beta x y}{1+\alpha y} \frac{y^{*}}{y} \\
& +\frac{e+\delta}{e q+\delta}\left(\frac{q \beta x^{*} y^{*}}{1+\alpha y^{*}}+\delta w^{*}-p y^{*} z^{*}\right) \\
& -\frac{e+\delta}{e q+\delta} \delta w^{*} \frac{w}{w^{*}} \frac{y^{*}}{y}+\frac{e+\delta}{e q+\delta} p y^{*} z \\
& +\frac{p(e+\delta)}{c(e q+\delta)}\left(-b z-c y z^{*}+b z^{*}\right)
\end{aligned}
$$




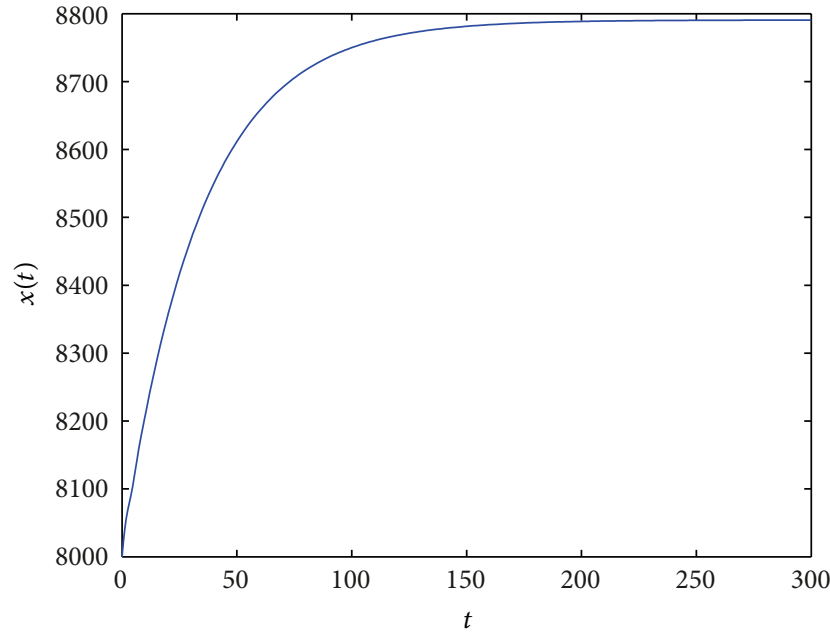

(a)

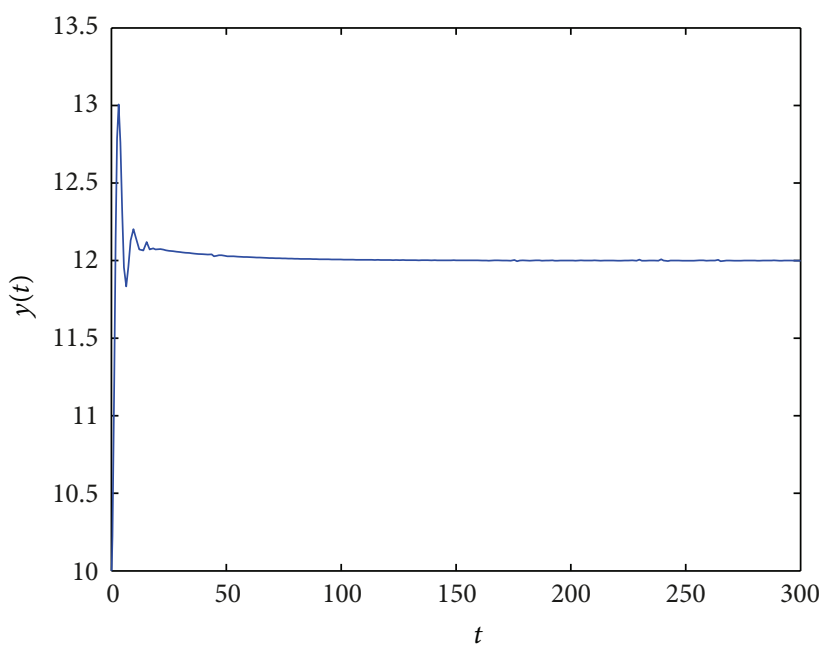

(c)

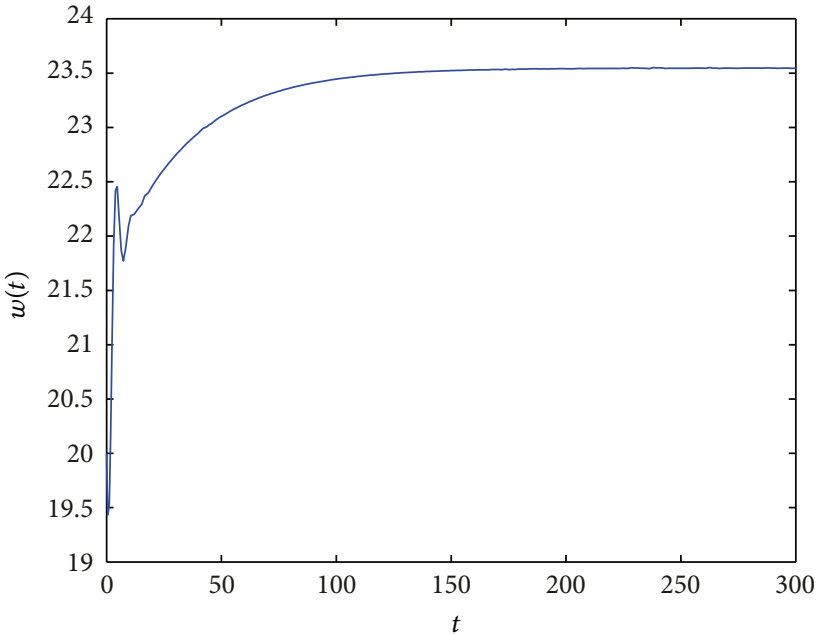

(b)

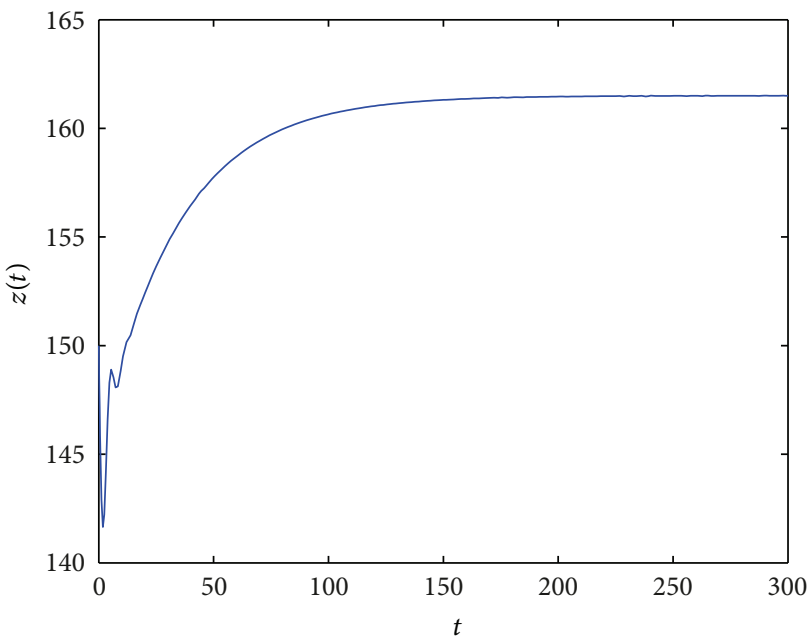

(d)

FIGURE 1: When $\tau=0.2<\tau_{0}$, the CTL-activated infection equilibrium $E^{*}(8790.7081,23.5465,12.0000,161.5044)$ of system (2) is asymptotically stable.

$$
\begin{aligned}
= & -\frac{d\left(x-x^{*}\right)^{2}}{x} \\
& -\frac{\alpha \beta x^{*}\left(y-y^{*}\right)^{2}}{\left(1+\alpha y^{*}\right)^{2}(1+\alpha y)}+\frac{\delta}{e q+\delta} \frac{(1-q) \beta x^{*} y^{*}}{1+\alpha y^{*}} \\
& \times\left(4-\frac{x^{*}}{x}-\frac{w^{*}}{w} \frac{1+\alpha y^{*}}{1+\alpha y} \frac{x y}{x^{*} y^{*}}-\frac{w}{w^{*}} \frac{y^{*}}{y}\right. \\
& \left.\quad-\frac{1+\alpha y}{1+\alpha y^{*}}\right)+\frac{e+\delta}{e q+\delta} \frac{q \beta x^{*} y^{*}}{1+\alpha y^{*}} \\
& \times\left(3-\frac{x^{*}}{x}-\frac{1+\alpha y^{*}}{1+\alpha y} \frac{x}{x^{*}}-\frac{1+\alpha y}{1+\alpha y^{*}}\right) .
\end{aligned}
$$

Noting that $x^{*}, w^{*}, y^{*}, z^{*}>0$, by $(61)$, we have that $\dot{W}^{*}(t) \leq$ 0 . Also, it is easy to verify that $\dot{W}^{*}(t)=0$ if and only if $x=x^{*}$, $w=w^{*}$, and $y=y^{*}$. We therefore derive from the third equation of system (2) that

$$
0=\dot{y}(t)=\frac{q \beta x^{*} y^{*}}{1+\alpha y^{*}}-a y^{*}+\delta w^{*}-p y^{*} z,
$$

which yields $z=z^{*}$. The maximal compact invariant set in $\left\{(x, \omega, y, v): \dot{W}^{*}(t)=0\right\}$ is the singleton $\left\{E^{*}\right\}$ when $R_{1}>$ 1 . Accordingly, the global asymptotic stability of $E^{*}$ follows. This completes the proof.

\section{Numerical Simulations and Conclusions}

In the following, we give examples to illustrate the main theoretical results above. In system (2), we choose a set of parameters as follows: $\lambda=270, d=0.02, \beta=0.001$, $\alpha=0.01, q=0.8, e=0.3, \delta=0.5, a=0.8, p=0.04$, $c=0.025$, and $b=0.3$. The initial values are $x_{0}=8000$, $w_{0}=20, y_{0}=10$, and $z_{0}=150$. Then $R_{1} \approx 9.0752>1$, 


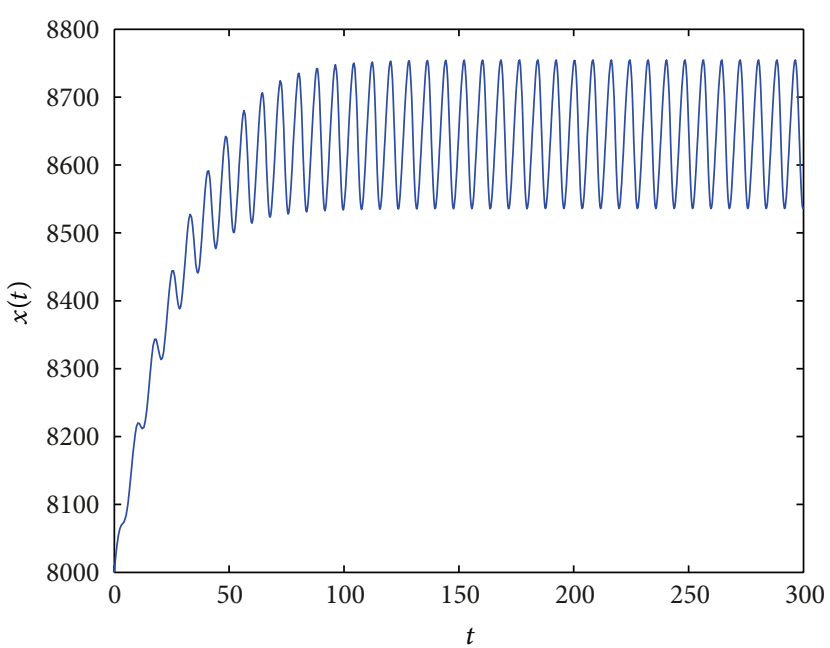

(a)

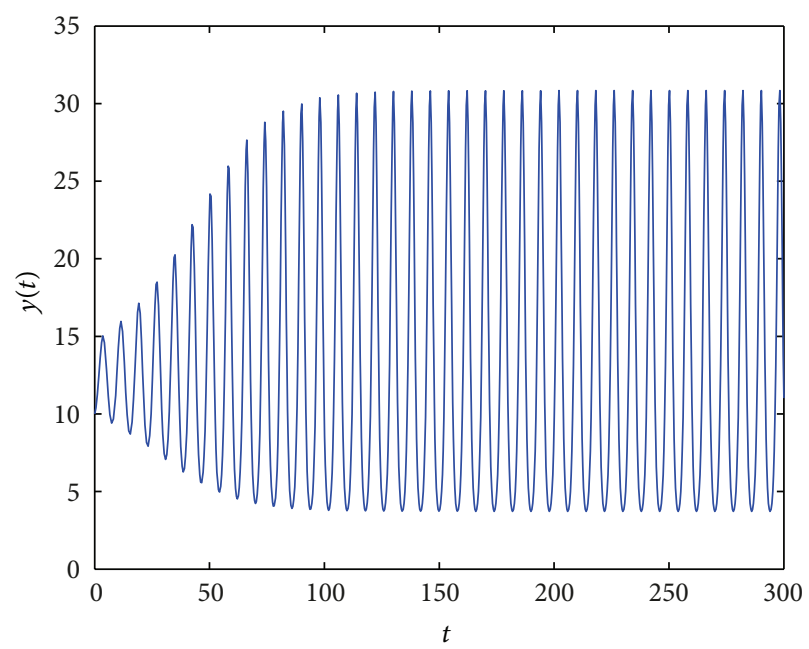

(c)

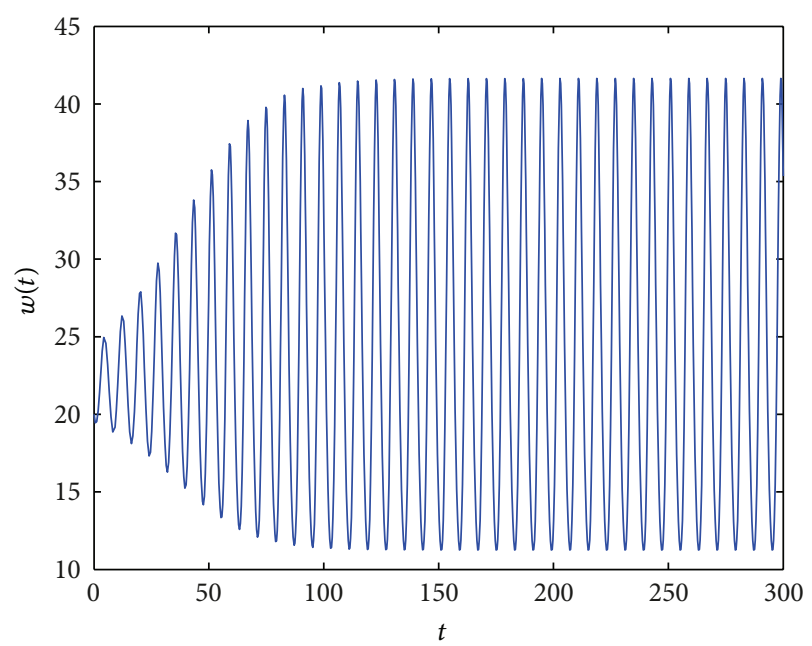

(b)

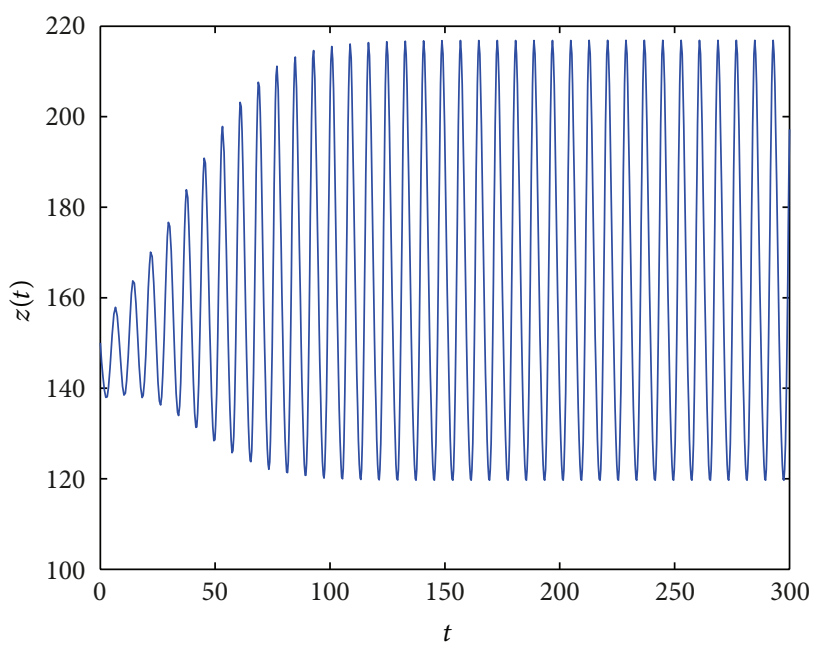

(d)

Figure 2: When $\tau=1.2>\tau_{0}$, the CTL-activated infection equilibrium $E^{*}(8790.7081,23.5465,12.0000,161.5044)$ of system (2) becomes unstable.

and the CTL-activated infection equilibrium $E^{*}$ exists. The values of these parameters satisfy the condition of Theorem 6 . By calculation, we derive that $\mu_{0} \approx 0.2860$ and $\tau_{0} \approx$ 0.3746 .

When $\tau=0.2<\tau_{0}$, the trajectory converges to the CTL-activated infection equilibrium $E^{*}$ (see Figure 1). While, when $\tau=1.2>\tau_{0}$, the CTL-activated infection equilibrium $E^{*}$ of (2) becomes unstable (see Figure 2), and the Hopf bifurcation occurs.

In this paper, we have investigated the global dynamics of an HIV-1 infection model with latently infected cells and delayed immune response. By giving the explicit expressions of two basic reproduction ratios $R_{0}$ and $R_{1}$, we first gave sufficient conditions for the existence of each of feasible equilibria of system (2). Then, a detailed analysis on the local asymptotic stability of the equilibria was carried out. It was shown that, if $R_{0}<1$ (hence, $E_{1}$ and $E^{*}$ are not feasible), the infectionfree equilibrium $E_{0}$ is locally asymptotically stable. If $R_{1}<$ 1 , the CTL-inactivated infection equilibrium $E_{1}$ is locally asymptotically stable. If $R_{1}>1$, the CTL-activated infection equilibrium $E^{*}$ exists. By giving Theorem 6 , we studied the existence of Hopf bifurcations at $E^{*}$. By constructing suitable Lyapunov functionals and using LaSalle's invariance principle, we proved that, for any time delay $\tau \geq 0$, if $R_{0}<1$, the infection-free equilibrium $E_{0}$ is globally asymptotically stable. If $R_{0}>1$ and $R_{1}<1$, the CTL-inactivated infection equilibrium $E_{1}$ is globally asymptotically stable. From this we can see that time delay has no effect on the stability of the infection-free equilibrium and the CTL-inactivated infection equilibrium. When $\tau=0$, we also proved the global asymptotic stability of the CTL-activated infection equilibrium $E^{*}$ of system (2) whenever it exists. 


\section{Acknowledgment}

This work was supported by the National Natural Science Foundation of China (nos. 11371368 and 11071254).

\section{References}

[1] M. A. Nowak and C. R. M. Bangham, "Population dynamics of immune responses to persistent viruses," Science, vol. 272, no. 5258, pp. 74-79, 1996.

[2] A. S. Perelson and P. W. Nelson, "Mathematical analysis of HIV1 dynamics in vivo," SIAM Review, vol. 41, no. 1, pp. 3-44, 1999.

[3] A. Korobeinikov, "Global properties of basic virus dynamics models," Bulletin of Mathematical Biology, vol. 66, no. 4, pp. 879-883, 2004.

[4] X. Song and A. U. Neumann, "Global stability and periodic solution of the viral dynamics," Journal of Mathematical Analysis and Applications, vol. 329, no. 1, pp. 281-297, 2007.

[5] X. Wang and X. Song, "Global stability and periodic solution of a model for HIV infection of CD4+ T cells," Applied Mathematics and Computation, vol. 189, no. 2, pp. 1331-1340, 2007.

[6] K. Wang, W. Wang, H. Pang, and X. Liu, "Complex dynamic behavior in a viral model with delayed immune response," Physica D, vol. 226, no. 2, pp. 197-208, 2007.

[7] A. A. Canabarro, I. M. Gléria, and M. L. Lyra, "Periodic solutions and chaos in a non-linear model for the delayed cellular immune response," Physica A, vol. 342, no. 1-2, pp. 234-241, 2004.

[8] H. Zhu, Y. Luo, and M. Chen, "Stability and Hopf bifurcation of a HIV infection model with CTL-response delay," Computers and Mathematics with Applications, vol. 62, no. 8, pp. 3091-3102, 2011.

[9] Z. Wang and R. Xu, "Stability and Hopf bifurcation in a viral infection model with nonlinear incidence rate and delayed immune response," Communications in Nonlinear Science and Numerical Simulation, vol. 17, no. 2, pp. 964-978, 2012.

[10] D. C. Douek, J. M. Brenchley, M. R. Betts et al., "HIV preferentially infects HIV-specific CD4+ T cells," Nature, vol. 417, no. 6884, pp. 95-98, 2002.

[11] O. Bagasra and R. J. Pomerantz, "Human immunodeficiency virus type I provirus is demonstrated in peripheral blood monocytes in vivo: a study utilizing an in situ polymerase chain reaction," AIDS Research and Human Retroviruses, vol. 9, no. 1, pp. 69-76, 1993.

[12] M. J. Pace, L. Agosto, E. H. Graf, and U. O'Doherty, "HIV reservoirs and latency models," Virology, vol. 411, no. 2, pp. 344354, 2011.

[13] D. C. Krakauer and M. Nowak, "T cell induced pathogenesis in HIV: bystander effects and latent infection," Proceedings of the Royal Society B, vol. 266, no. 1423, pp. 1069-1075, 1999.

[14] M. A. Capistrán, "A study of latency, reactivation and apoptosis throughout HIV pathogenesis," Mathematical and Computer Modelling, vol. 52, no. 7-8, pp. 1011-1015, 2010.

[15] J. K. Hale and S. Verduyn Lunel, Introduction to Functional Differential Equations, Springer, New York, NY, USA, 1993.

[16] J. P. LaSalle, The Stability of Dynamical System, Regional Conference Series in Applied Mathematics, SIAM, Philadephia, Pa, USA, 1976. 


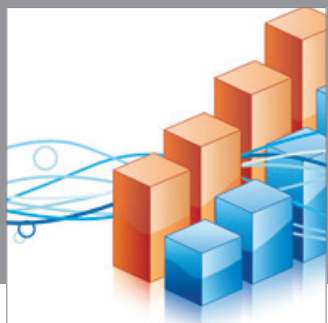

Advances in

Operations Research

mansans

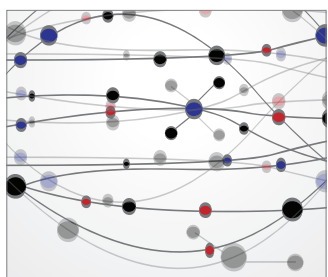

The Scientific World Journal
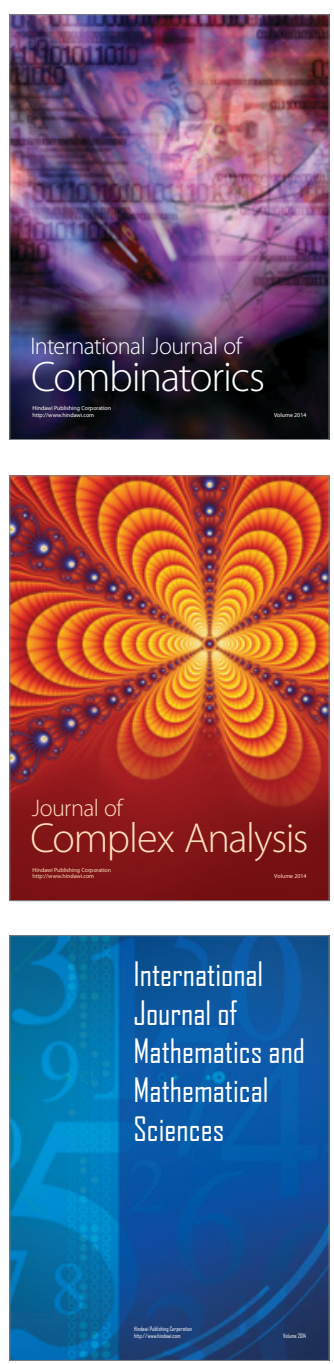
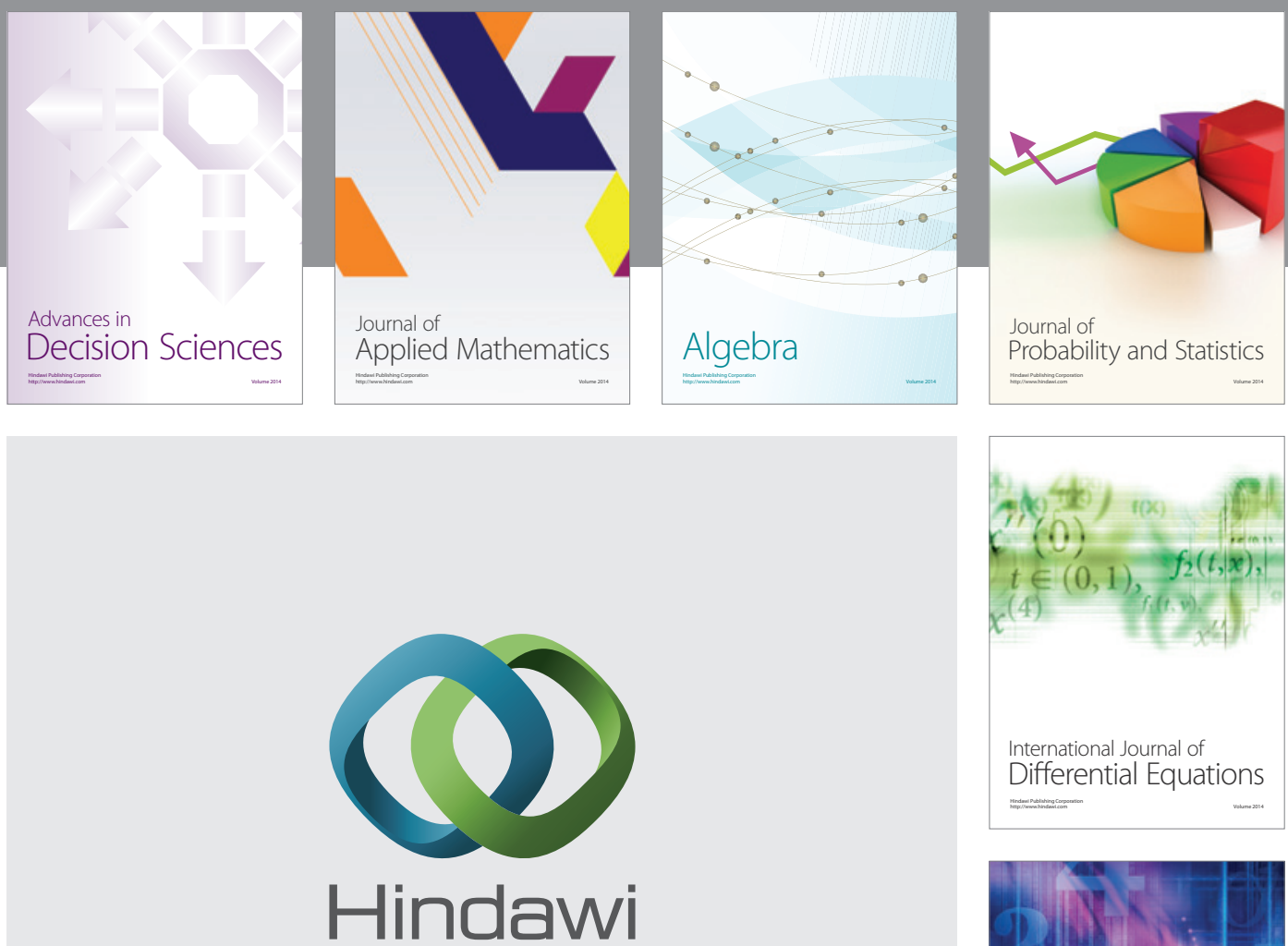

Submit your manuscripts at http://www.hindawi.com
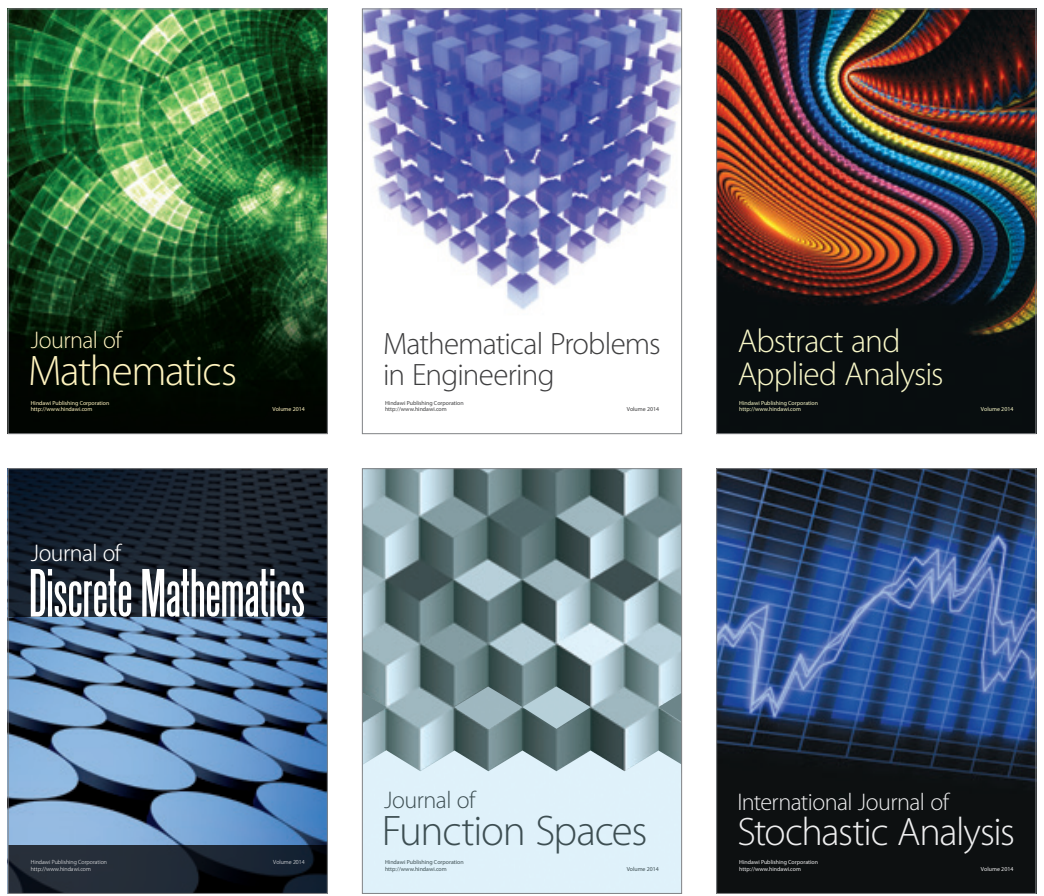

Journal of

Function Spaces

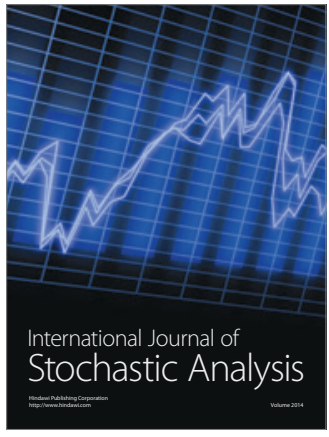

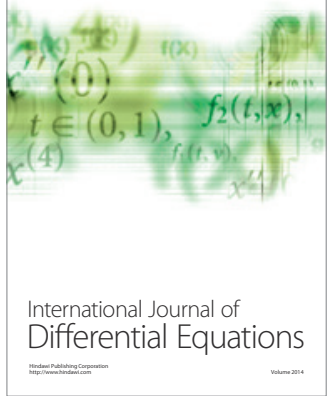
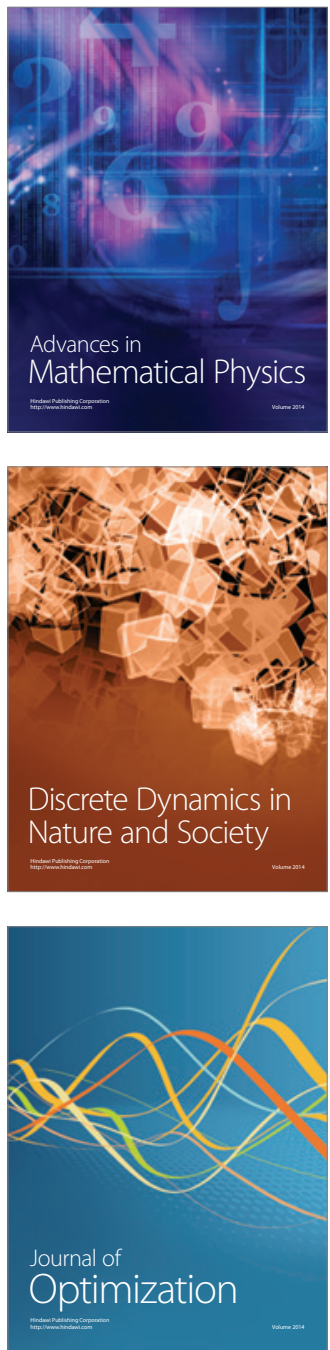